UCRL-JC-123479

PREPRINT

\title{
A Rapid Supercritical Extraction Process for the Production of Silica Aerogels
}

\author{
J.F. Poco
}

P.R. Coronado

R.W. Pekala

L.W. Hrubesh

This paper was prepared for submittal to the Materials Research Society 1996 Spring Meeting

San Francisco, CA

April 8-12, 1996

April 1996

This is a preprint of a paper intended for publication in a journal or procecdings. Since changes may be made before publication, this preprint is made available with the understanding that it will not be cited or reproduced without the permission of the author. 


\section{DISĆLAIMER}

This document was prepared as an account of work. sponsored by an agency of the United States Government. Neither the United States Government nor the University of California nor any of their employees, makes any warranty, express or implied, or assumes any legal liability or responsibility for the accuracy, completeness, or usefulness of any information, apparatus, product, or process disclosed, or represents that its use would not infringe privately owned rights. Reference herein to any specific commercial product, process, or service by trade name, trademark, manufacturer, or otherwise, does not necessarily constitute or imply its endorsement, recommendation, or favoring by the United States Government or the University of California. The views and opinions of authors expressed herein do not necessarily state or reflect those of the United States Government or the University of California, and shall not be used for advertising or product endorsement purposes. 


\section{DISCLAMMER}

Portions of this document may be illegible in electronic image products. Images are produced from the best available original document. 


\title{
A RAPID SUPERCRITICAL EXTRACTION PROCESS FOR THE PRODUCTION OF SILICA AEROGELS*
}

\author{
J.F. POCO, P.R. CORONADO, R.W. PEKALA and L.W. HRUBESH, Chemistry \& \\ Materials Science Department, Lawrence Livermore National Laboratory, Livermore, CA \\ 94550 hrubesh1@llnl.gov \\ * Work performed under the auspices of the U.S. Department of Energy by the Lawrence \\ Livermore National Laboratory under Contract No. W-7405-ENG-48.
}

\begin{abstract}
Silica aerogels are a special class of porous materials in which both the pore size and interconnected particle size have nanometer dimensions. This structure imparts unique optical, thermal, acoustic, and electrical properties to these materials. Transmission electron microscopy and small angle $\mathrm{x}$-ray scattering show that this nanostructure is sensitive to variations in processing conditions that influence crosslinking chemistry and growth processes prior to gelation. Recently, Lawrence Livermore National Laboratory (LLNL) has demonstrated that a Rapid Supercritical Extraction (RSCE) process can be used to prepare near-net shape silica aerogels in hours rather than days. Preliminary data from RSCE silica aerogels show that they have improved mechanical properties and slightly lower surface areas than their conventionally dried counterparts, while not compromising their optical and thermal performance.
\end{abstract}

\section{INTRODUCTION}

Aerogels are a special class of microporous materials which are derived from highly crosslinked inorganic or organic sols. During their chemical synthesis, the crosslinked sols form a gel which is a solid network surrounded by a liquid. An aerogel results when the liquid is removed from the gel using special techniques to prevent collapsing the delicate structure, then replacing the liquid with air. Aerogels have a unique nanostructure consisting of particles and pores which are smaller than wavelengths of visible light. Such a fine nanostructure is responsible for many of the exceptional properties exhibited by aerogels, such as, the lowest thermal conductivity, refractive index and dielectric constant of any known solids [1]. However, the fine nanostructure is also the reason that aerogels are difficult to produce in monolithic form. The aerogels have such small pores (i.e., $<100 \mathrm{~nm}$ ) that they cannot be dried quickly by evaporation without shrinking and cracking, primarily due to tremendous surface tension forces within the porous structure [2]. For insight into the problems associated with drying aerogels, the reader is referred to an excellent review article by Scherer [3] on the subject of the physics of drying gels.

A method to circumvent the problem of large capillary forces, which is used to produce aerogels commercially, is to heat the liquid-filled gel under pressure until the liquid converts to a supercritical fluid. In it's supercritical fluid state, the liquid no longer has surface tension and the fluid can be directly removed from the gel without cracking it. Normally, this process must be done slowly, so that the fluid expansion due to the increasing temperature does not stress and crack the gel $[4,5]$. This process, called Supercritical Extraction (SCE), typically requires many hours to complete and it is costly. Considerable work has been done by several authors, to analyze and understand the supercritical drying process in aerogels, with the intent to eliminate or at least minimize cracking, and to speed-up the process $[6,7,8]$. The Rapid Supercritical Extraction (RSCE) process described in this paper, was developed to speed the production of alkoxide derived aerogels and to be able to form precise net-shapes. This process does not require formation of the gel before drying and it minimizes the stresses in the 
gel during conversion of the liquid to the supercritical state; thus it speeds the entire process of making aerogels, start-to-finish.

\section{EXPERIMENTAL}

\section{The Concept of Gel Containment During Drying}

The supercritical extraction process for aerogels requires that the gel is heated, under pressure, until the critical temperature, $T_{c}$, is reached. Scherer, in one of his many papers on the subject [5], identifies the differential strain between the pore liquid and the gel network as the source of stresses in the heated gel. These stresses are the result of differences in the differential thermal expansion coefficients between the liquid and the solid phases of the gel, and from the shrinkage of the gel due to syneresis. Thus, when the gel is not constrained, rapid heating will expand the liquid faster than the solid network thermally expands, which stretches the gel network in those directions where the liquid leaves the gel. At the same time, syneresis causes a contraction of the gel network, exacerbating the stresses. If the gel is constrained in any direction, the strains are enhanced in the other non-constrained directions, which may also increase the stresses. The gel suffers cracks when the differential stresses exceed the basic strength of the gel (i.e., it's modulus of rupture [9]).

The strains and the associated stresses during heating, can be minimized by containing the gel entirely within a strong mold body. In this case, the 'positive' strain due to expanding liquid is essentially zero, except at the mold outlet where it will be highly localized. The 'negative' strain (shrinkage) due to syneresis, still has to be accounted for, but it can be minimzed by rapid heating to reduce the time the gel is wet. Our process uses the concept of total gel containment and rapid heating, to produce monolithic aerogels. The process has an added advantage of speeding aerogel production by allowing the formation of the gel directly within the mold while it is being heated. Also, the use of metal molds in this process allows the ability to make near net-shape aerogels and precision surfaces. This process has been found to work well for several varieties of alkoxide derived gels, and it is useful for both polymeric and colloidal aerogel types.

\section{Process Description}

The RSCE process is very similar to injection molding, a common process used to manufacture certain types of plastics. In this process, the precursor chemicals (liquids) are injected directly into a two-piece, sealed mold, having connecting tubing. The mold is rapidly heated, during which time the liquids react to form the gel within the mold. As heating continues, the liquid trapped within the gel expands to create a hydrostatic pressure within the mold, while some liquid leaks out through an exit port. After a short time, both the temperature and pressure within the mold exceed the critical point of the liquid and it becomes a supercritical fluid. The fluid is removed from the delicate gel network through an opening in the mold by rapid decompression and then the heaters are de-energized. After the mold is sufficiently cooled, it is opened and the finished aerogel part is removed.

The entire process, i.e., filling the mold, gelation, heating to the critical point of the entrained liquid, decompression of the supercritical fluid, cooling the mold and disassembly, is typically accomplished in less than $3 \mathrm{hr}$., but it can be done in less than $1 \mathrm{hr}$. To date, we have used this process to make monolithic pieces of silica aerogels as large as $20 \mathrm{~cm}$ diameter by $3 \mathrm{~cm}$ thick. We believe that this process is scaleable to much larger sizes. 


\section{Gel Precursor Preparation}

The precursors used in the RSCE process are prepared by routine sol-gel chemistry, using metal alkoxides. For example, we use tetramethoxysilane (TMOS) for the silica aerogels; mixing TMOS, methanol, water, and catalyst in either a single-step or a two-step procedure, as previously described [10]. The precursors are typically prepared within $1 \mathrm{hr}$. of the starting the process. The precursor liquid is loaded into a syringe and then injected into the mold through the connecting tubing; alternatively, the precursor is poured into the mold to overflowing, and the cover is placed on the mold to seal in the precursor fluid.

\section{Functional Description}

Conventional direct supercritical extraction (SCE) is accomplished using a pressure vessel (autoclave) large enough to hold parts of appreciable size. The procedure is as follows: the pre-formed gel, typically in a glass mold, is placed within the autoclave vessel. The mold has at least one open end. The remaining volume in the vessel is filled with alcohol; this covers the wet gel to preclude evaporative drying, and it also provides the hydrostatic pressure needed to prevent boiling as the temperature is raised. The vessel is sealed. The heaters are energized and temperature of the vessel is programmed to increase at a rate of $0.3^{\circ} \mathrm{C}$ per minute (this rate is slow enough to prevent cracking, in most cases). The hydrostatic pressure within the vessel rises rapidly to 100 bars; excess pressure is relieved through a valve. After critical conditions for the alcohol have been exceeded (i.e., $T \approx 250{ }^{\circ} \mathrm{C}, \mathrm{P} \approx 80$ bars), the temperature is held constant while the pressure is released from the vessel at a rate of about 0.3 bar per minute. The heaters are de-energized after the pressure reaches $1 \mathrm{bar}$. The vessel is purged with dry air as it cools to ambient temperature, completing the process.

The rapid supercritical extraction process (RSCE) differs from the conventional process in several respects; the hardware is simpler and less costly, the gels do not have to be preformed, and the RSCE process is much faster. A functional description of the process is given with reference to the diagram in Fig.1. A two piece mold (TPM) is used in the RSCE process. The mold pieces fit together so that a metal-metal seal is formed and bolts are used to fasten them together. The molds may also have a small port with tube connected to it. A heating coil (HC) is wrapped around the assembled mold and the mold is placed within a vacuum chamber (VC). The purpose of the vacuum containment is to provide thermal insulation for the heated mold and to prevent explosive hazard by containing alcohol in case of a leaky mold. The vacuum chamber is sealed and evacuated to about 0.001 bar pressure with the vacuum pump (VP). The mold is filled by injecting the precursor fluid through valve (V1) and the connecting tubing. The heater is then energized and the temperature increases at a rate of $20^{\circ} \mathrm{C}$ per minute. The precursor liquid within the mold expands, creating hydrostatic pressure (P1) through the connecting tubing, to a relief valve (RV). The relief valve (RV) allows excess pressure above its set-point ( 100 bars) to be expelled to an external container. After the critical temperature of alcohol is reached, the pressure within the mold is released through the valve (V1) at a rate of 5 bars per minute, until the pressure decreases to about 1 bar. The remaining atmosphere is removed by vacuum. The heaters are de-energized and the mold is allowed to cool before it is removed and disassembled. The entire process to make an aerogel, can be accomplished in 60 minutes, from start (liquid precursor) to finish (dry aerogel). The conventional process requires a minimum of 30 hours, in addition to the time required for the gel to form in the mold prior to drying (gel time varies from minutes to weeks, depending on the targeted density). A comparison of the time-temperature-pressure profiles is shown in Fig. 2 for typical RSCE and SCE processes. 


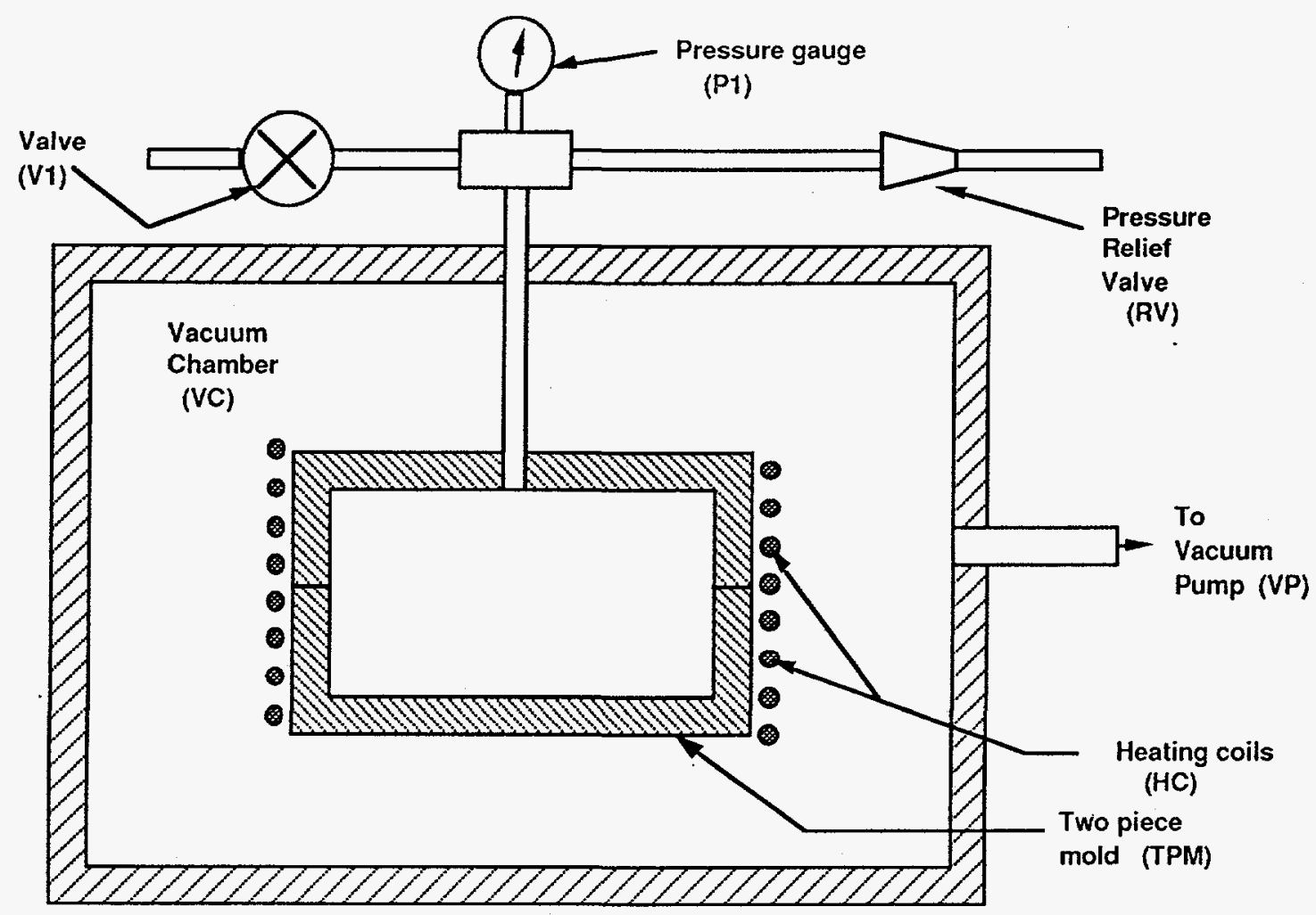

Figure 1. Schematic diagram showing main components of RSCE apparatus.

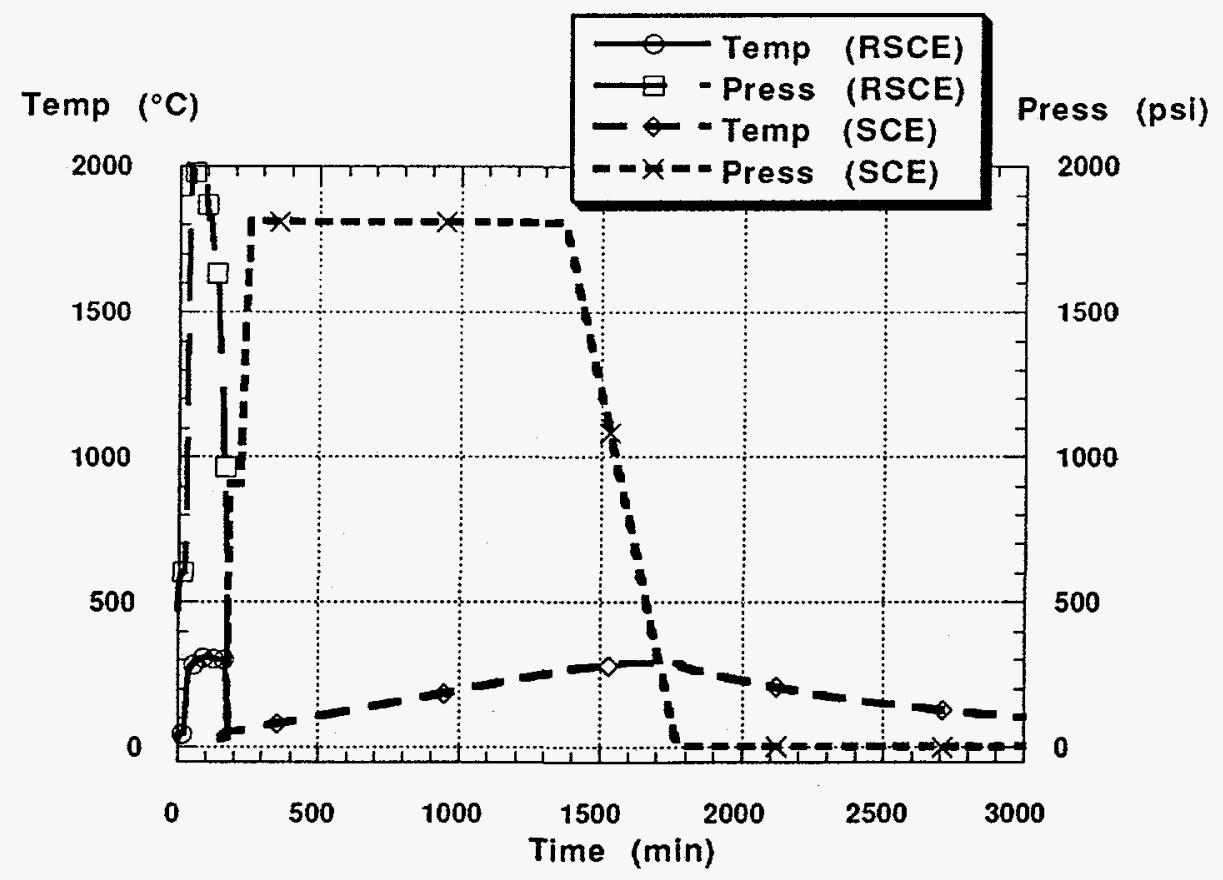

Fig. 2. Time-temperature-pressure plots showing that the RSCE process is $10 \mathrm{x}$ faster than the SCE process. 
An alternative mode for RSCE, requiring a secondary pressure vessel (autoclave), is also described. For this case, the precursor liquids are poured into two-piece molds which are assembled and sealed. The sealed molds (without a connecting tube or heating coils) are placed into the pressure vessel. The remaining volume of the autoclave is filled with alcohol before it is sealed. The heaters to the autoclave are energized to raise the temperature at a rate of $5^{\circ} \mathrm{C}$ per minute. The internal pressures of both the autoclave vessel and the sealed molds within it, rise due to liquid expansion. Excess pressure in the autoclave vessel is released through a relief valve into another container. The hydrostatic pressure of the fluid external to the mold increases faster than that inside the mold because it heats and expands faster. The gel forms within the mold during this time. At some point during the heating, the pressure inside the mold becomes sufficiently higher than the external pressure and the alcohol leaks out of the mold into the surrounding liquid. After the critical temperature of the alcohol is reached, the pressure of the liquid in the autoclave is released at a rate of 3 bars per minute, until it is reduced to about 1 bar. The heaters are then de-energized and the autoclave vessel is purged with dry air (or evacuated) while it cools to ambient temperature. The autoclave vessel is opened and the molds are removed and disassembled.

\section{RESULTS AND DISCUSSION}

\section{$\underline{\text { RSCE compared to conventional SCE }}$}

The RSCE process can produce aerogels, from start-to-finish, 30 times faster than existing supercritical drying processes because the gel forms during the process and the liquids can be rapidly purged from the confined gel without cracking it. The RSCE process can produce aerogels having precise sizes and shapes, maintaining high surface tolerances. This is possible because the mold totally defines the size, shape, and surface quality of the aerogel object. The RSCE process reduces liquid waste by $40 \%$ of that produced by conventional SCE because the liquid within the gel is purged before decomposing and it can be re-used to make more aerogels. The RSCE process uses about 3 times less energy than other methods because it does not require pumping fluids and the heating of the smaller molds for shorter times, is more energy efficient. We estimate that the production cost per unit can be 8 times less for RSCE than the same unit by conventional processes, because of the rapid production rates that are possible.

\section{Properties of RSCE silica aerogels}

The silica aerogels produced by the RSCE process exhibit higher strengths and lower surface areas, than those produced by SCE using the same gel precursors. Fig. 3 shows the elastic modulus (measured by sound velocity) versus density, for silica aerogels made by the two different processes. The scaling factors for the two sets of data are similar $(\sim 2.6)$, but the RSCE aerogels have a factor of 3 higher modulus than the SCE aerogels. The BET surface areas for the RSCE silica aerogels for densities in the range $110-330 \mathrm{~g} /$, average $536 \mathrm{~m}^{2} / \mathrm{g}$ compared with an average of $726 \mathrm{~m}^{2} / \mathrm{g}$ for SCE silica aerogels. These data suggest that the RSCE process produces aerogels having larger particles and larger necks between particles; probably the result of accelerated reactions due to gelation occuring at high temperatures, compared to the ambient temperature gels produced by SCE.

Preliminary measurements of the thermal conductivity for representative RSCE aerogels, show that they are comparable to the SCE aerogels having the same densities. Also, the optical clarity of the RSCE aerogels is qualitatively the same as the SCE aerogels. 


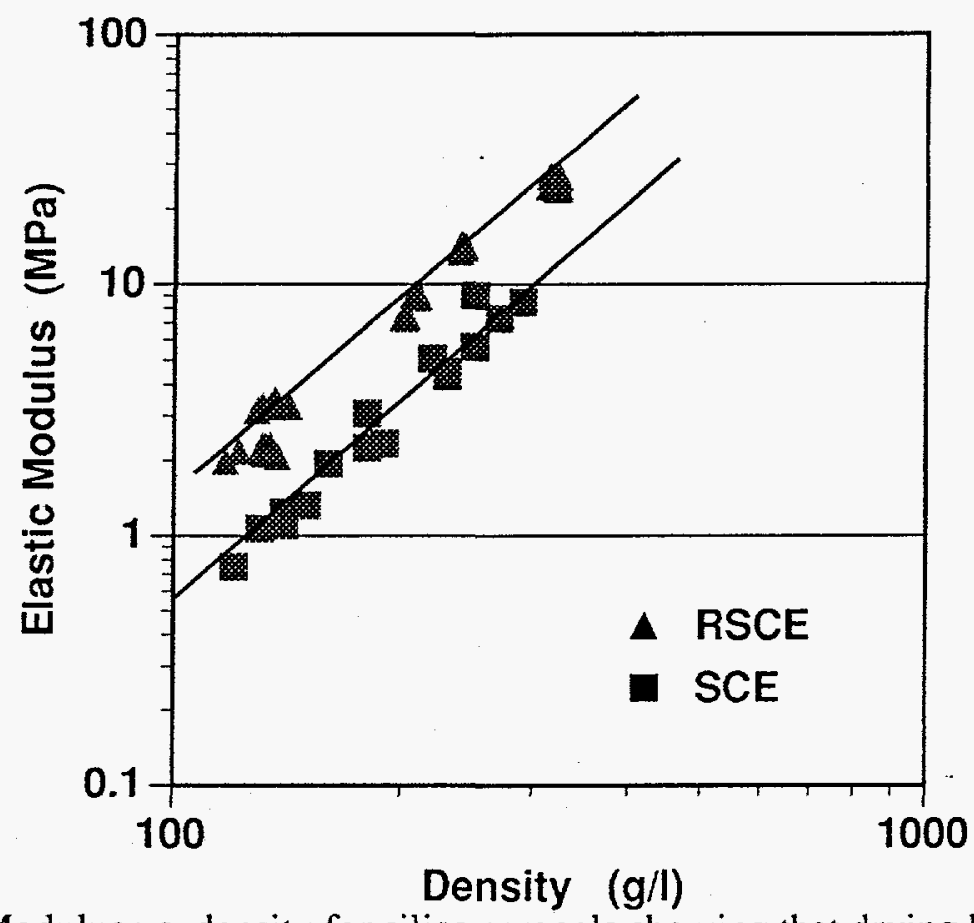

Figure 3. Modulus vs. density for silica aerogels showing that drying by RSCE produces stronger aerogels compared with conventional SCE drying.

\section{CONCLUSIONS}

We have developed a rapid supercritical drying process that significantly speeds the preparation of aerogels derived from alkoxides. Aerogels can be made from start-to-finish, in less than 1 hour using this process. The aerogels can be made to near net-shapes, with high tolerance surface finishes. The aerogels are characteristically 3 times stronger than those made by conventional SCE drying, probably because they consist of larger silica particles. This method is energy efficient and reduces waste, and therefore it is less costly than conventional SCE processes. These clear advantages of the new process over existing drying processes, should help stimulate the commercialization of aerogel materials.

\section{References:}

1) J.Fricke and A.Emmerling, in Chemistry, Spectroscopy, and Applications of Sol-Gel Glasses, edited by R. Reisfeld and C.K.Jorgensen, (Springer Series on Structure and Bonding, Vol. 77, Springer-Verlag, Heidelberg, Germany, 1991), p.37.

2) G.W.Scherer, J. Am. Ceram. Soc. 69, 473 (1986).

3) G.W.Scherer, in Ultrastructure Processing of Advanced Materials, edited by D.R. Uhlmann and D.R.Ulrich, (J. Wiley \& Sons, N.Y., 1992), p.181.

4) C.J.Brinker and G.W.Scherer, Sol-Gel Science (Acad. Press, N.Y., 1990), p.413.

5) G.W.Scherer, J. Non-Cryst. Solids 145, 33 (1992).

6) G.W.Scherer, H.Hdach and J.Phalippou, J. Non-Cryst. Solids 130, 157 (1991).

7) G W.Scherer, J. Sol-Gel Science Science and Technology 3, 127 (1994); T.Woignier, G.W.Scherer and A.Alaoui, ibid., p.141.

8) J. Phalippou, G.W.Scherer, T.Woignier, D.Bourret and R.Sempe're', J. Non-Cryst. Solids 186, 64 (1995); M.J.vanBommel and A.B.deHaan, ibid., p.78.

9) G.W.Scherer, S.A.Pardenek, and R.M.Swiatek, J. Non-Cryst. Solids 107, 14 (1988).

10) L.W.Hrubesh, T.M.Tillotson, and J.F.Poco, in Chemical Processing of Advanced Materials, edited by L.L.Hench and J.K.West, (J. Wiley \& Sons, N.Y., 1992), p.19. 